\section{TOTAL PROTECTION FOR YOUR PRACTICE}

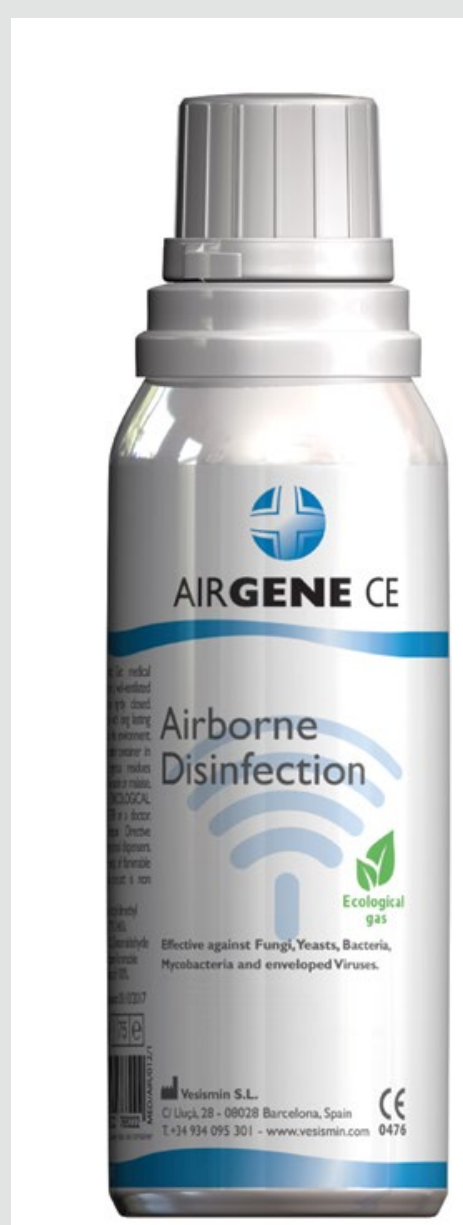

When it comes to the transmission of harmful pathogens, dental practices are one of the most contaminated working environments in the world. The risk of cross infection is particularly high due to the dispersal of aerosols within the surgery caused by the use of high speed turbines in common treatments.

AIRGENE CE Airborne Disinfectant from Eschmann helps minimise this risk, enhancing existing hygiene processes and only taking one hour to ensure a 'belt and braces' approach to the daily decontamination regime.

AIRGENE CE's 'one shot' valve which automatically releases the high-level disinfectant achieves a dual effect: effective decontamination of micro-organisms over a wide area and elimination of bad odours caused by bacterial decomposition.

Just one $50 \mathrm{ml}$ canister covers an area of up to $40 \mathrm{~m}^{3}$, treating all surfaces, especially hard to reach and inaccessible places.

For use in dental surgeries and patient areas, AIRGENE CE should be used on a weekly basis or as frequently as required.

For more information on the highly effective and affordable range of decontamination equipment and products from Eschmann, visit www.eschmann.co.uk or call 01903875787.

\section{ONE SMALL CHANGE}

Pärla Toothpaste Tabs offer an ethical, plastic-free solution to conventional toothpaste.

The dehydrated tabs come in a reusable glass jar - to use they are simply chewed for five seconds and then used for brushing as normal with a wet toothbrush. Developed by practising dentists - Simon Chard, Rhona Eskander and Adarsh Thanki - you can rest assured that the product does not compromise on oral health benefits either. SLS-free for reduced risk of irritation, Pärla contains fluoride to protect against decay. Pärla Toothpaste Tabs are:

- Plastic-free

- Animal cruelty-free

- Palm oil-free

- SLS-free

- Gluten-free

- Made in the UK

- Vegan

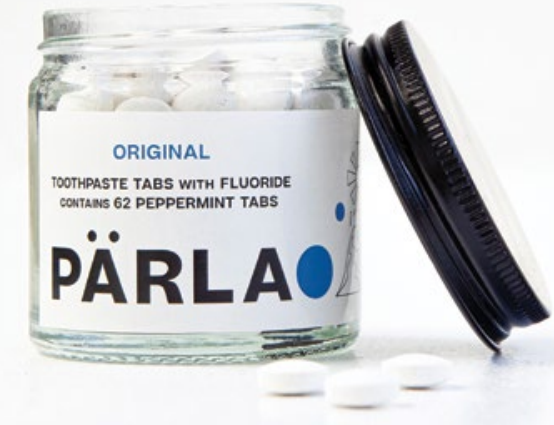

- Contain fluoride.

Help your patients make one small change to help protect the planet while maintaining their oral hygiene with innovative Pärla Toothpaste Tabs.

For more information, visit parlatoothpastetabs.com.

\section{REMOTE WORKING SOLUTIONS FOR YOUR PRACTICE}

Now more than ever, remote working solutions are vital. Yo Telecom is here to help.

Yo Telecom is setting up virtual receptions for dental practices. This will allow you and your staff to take calls, have telephone triage, and arrange emergency appointments. Yo Telecom will provide softphones on your mobiles and laptops, meaning you can make and take calls through the main number of your practice. You can also transfer calls to your dentists for emergency cases, and retrieve all answer phone messages left from patients.

You will also be able to access your dental database and reception computers from your home. Yo Telecom's remote access software enables you to use your practice's computers from anywhere in the world. This will ensure that during these times, your quality of service remains high and you have access to all of your patients' information.

Get in touch now to find out how Yo Telecom can help you on 02381733966 .

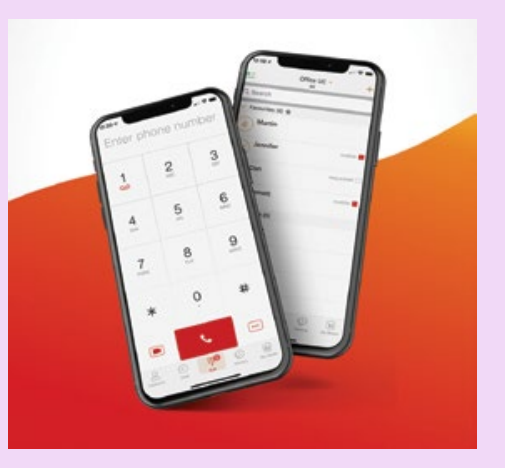

\section{GETTING BACK} ONTRACK

As dental practices start to reopen, this is going to be a very critical time for your dental practice, your team and of course, your patients. Henry Schein have produced a bounce back checklist with suggestions and ideas for how you can do this.

To find out more visit https://issuu. com/henryscheinuk/docs/hsd162-04-20 planning_your_business_bounceback_ fin?fr=sMzViZjExMDc5Mjc. 